\title{
Persisting Cough as the Single Presenting Symptom of an Intrathoracic Tumor in a Nine-Month-Old Child with Adenovirus Airway Infection
}

\author{
Wiegering $\mathrm{V}^{*}$, Schlegel $\mathrm{PG}^{1}$, Winkler $\mathrm{B}^{1}$, Lazarus $\mathrm{M}^{3}$, Wirth $\mathrm{C}^{4}$, Ernestus $\mathrm{K}^{5}$, Walles $\mathrm{T}^{6}$ and Liese $\mathrm{J}^{2}$ \\ ${ }^{1}$ University Children's Hospital Würzburg, Department of Pediatric Hematology / Oncology and Stem Cell \\ Transplantation, Germany \\ ${ }^{2}$ University Children's Hospital Würzburg, Department of Infectiology and Immunology, Germany \\ ${ }^{3}$ University Hospital Würzburg, Department of Anesthesia, Germany \\ ${ }^{4}$ University Hospital Würzburg, Division of Pediatric Radiology, Department of Radiology, Germany \\ ${ }^{5}$ University of Würzburg, Institute of Pathology, Germany \\ ${ }^{6}$ University Hospital Würzburg, Department of Thorax Surgery, University Medical Center ZOM Würzburg, Ger- \\ many
}

${ }^{*}$ Corresponding author: Wiegering V, MD, University Children's Hospital, Department of Pediatric Hematology / Oncology, D31, Josef-Schneider-Strasse 2, D-97080 Würzburg, Germany, Tel: 49-931-20127728, Fax: 49-931-201-27649,E-mail: Wiegering_V@ukw.de

Citation: Wiegering V, Schlegel PG, Winkler B, Lazarus M, Wirth C, et al. (2015) Persisting Cough as the Single Presenting Symptom of an Intrathoracic Tumor in a Nine-Month-Old Child with Adenovirus Airway Infection. J Case Rep Stud 3(2): 204. doi: 10.15744/2348-9820.2.504

Received Date: February 25, 2015 Accepted Date: April 01, 2015 Published Date: April 08, 2015

\begin{abstract}
We report on a nine-month-old girl who presented with persisting cough, and diminished ventilation of the left hemithorax. Viral pneumonia was suspected after Adenovirus detection by PCR, but chest X-rays showed a persistent shadowing of the left hemithorax and persistent coughing despite clinical improvement. Because of the discrepancy between clinical and radiological signs further investigations by ultrasound and CT scan were performed, which visualized an intrathroracic tumor. Histopathology confirmed diagnosis of a teratoma.

This case highlights the need for careful evaluation by the treating physicians. If the chest X-ray provides a discrepancy to the clinical findings or persistent pathologies exist, differential diagnosis should be discussed and further diagnostics be performed.
\end{abstract}

\section{Introduction}

Teratomas are germ cell tumors that arise in children from a variety of locations. They are considered tumors that originate from primordial cells with the potential to differentiate into many different types of tissue [1]. The most common sites in children include saccrococcygeal (40\%), ovary (25\%), testicle (12\%), brain (5\%) and rarely other locations [2]. In more than half of the patients mediastinal teratomas present with unspecific symptoms or are asymptomatic. Often, they are discovered on radiological evaluation by chance [3]. In the thoracic cavity they can be easily missed or may masquerade as pneumonic infiltrations. Reported complications are due to intrathoracic compression effects, rupture of the tumor or secretion of hormones. Early diagnosis and resection is favored. Here, we report on a nine-month-old girl that presented in our outpatient clinic with symptoms of an airway infection, and suspected pneumonia.

\section{Case Report}

A 9-month-old girl presented in the outpatient clinic with persistent cough for three weeks and subfebrile temperatures (max. $38.5^{\circ} \mathrm{C}$ ). No recurrent chest infections were observed in the medical history. On clinical examination there was no tachypnea $\left(\mathrm{SO}_{2}\right.$ 96\%), but diminished breath sounds were auscultated over her left thorax, the percussion was damped suitable for the clinical suspected pneumonia. Therefore, a chest X-ray was undertaken which revealed a large, homogenous shadowing of the left hemithorax with a mild shifting of the mediastinum (Figure 1A). The child was hospitalized with suspected pneumonia and treated accordingly with antibiotics. Adenovirus infection, however, could be detected by PCR of the nasopharyngeal aspirate. The clinical condition improved quickly, but the ipsilateral difference in auscultation persisted as well as the cough. Therefore, chest X-ray was repeated four days later and showed persistent left side opacities (Figure 1B). Because of the discrepancy of clinical and radiologic findings, further diagnostics were added and the working diagnosis of viral pneumonia was reevaluated. Thoracic ultrasound did not show an effusion, but revealed a paracardial consolidation (Figure 1C). Consecutively, low dose computed tomography of the chest was performed. It showed a large, well-defined heterogeneous mass in the left hemithorax $(6.5 \mathrm{x} 4.5 \mathrm{x} 8 \mathrm{~cm})$ evolving in the anterior 
upper mediastinum and extending into the pleural cavity (Figure 1E). The lesion showed areas of irregular nodularity, calcifications as well as fat attenuation along with soft tissue components. No signs of local infiltration or lymphadenopathy were seen. These features were considered suggestive of a mature teratoma. Laboratory evaluation including tumor markers (alpha fetoprotein, NSE, $\beta$-HCG) and catecholamine metabolites were within the normal limits.

En-bloc tumor resection was performed by way of a hemiclamshell incision of the left pleural cavity. Intraoperatively, a capsulated, circumscribed inhomogenous, white-grayish colored mass was found (Figure 1F). It was dissected from the mediastinal structures including portions of the thymus gland, taking care to preserve the left phrenic nerve, the diaphragm and the lung. The thoracic cavity was closed using resorbable sutures, and a chest tube was placed in order to initiate postoperative suction therapy to support re-expansion of the atelectatic left lung $\left(20 \mathrm{~cm} \mathrm{H}_{2} \mathrm{O}\right)$. The postoperative course was uneventful, the chest drain was removed on the second postoperative day and the chest control X-ray showed a normalized left thorax apparatus without postoperative complications (Figure 1D). Histopathological work-up confirmed a mature teratoma with immature neural elements. Further clinical and radiological (thoracic x-ray and postoperative MRI) follow-up showed complete remission without limitations of the lung capacity. The patient is currently doing well two years after resection without respiratory limitations or signs of a tumor relapse. Tumor markers remained negative.

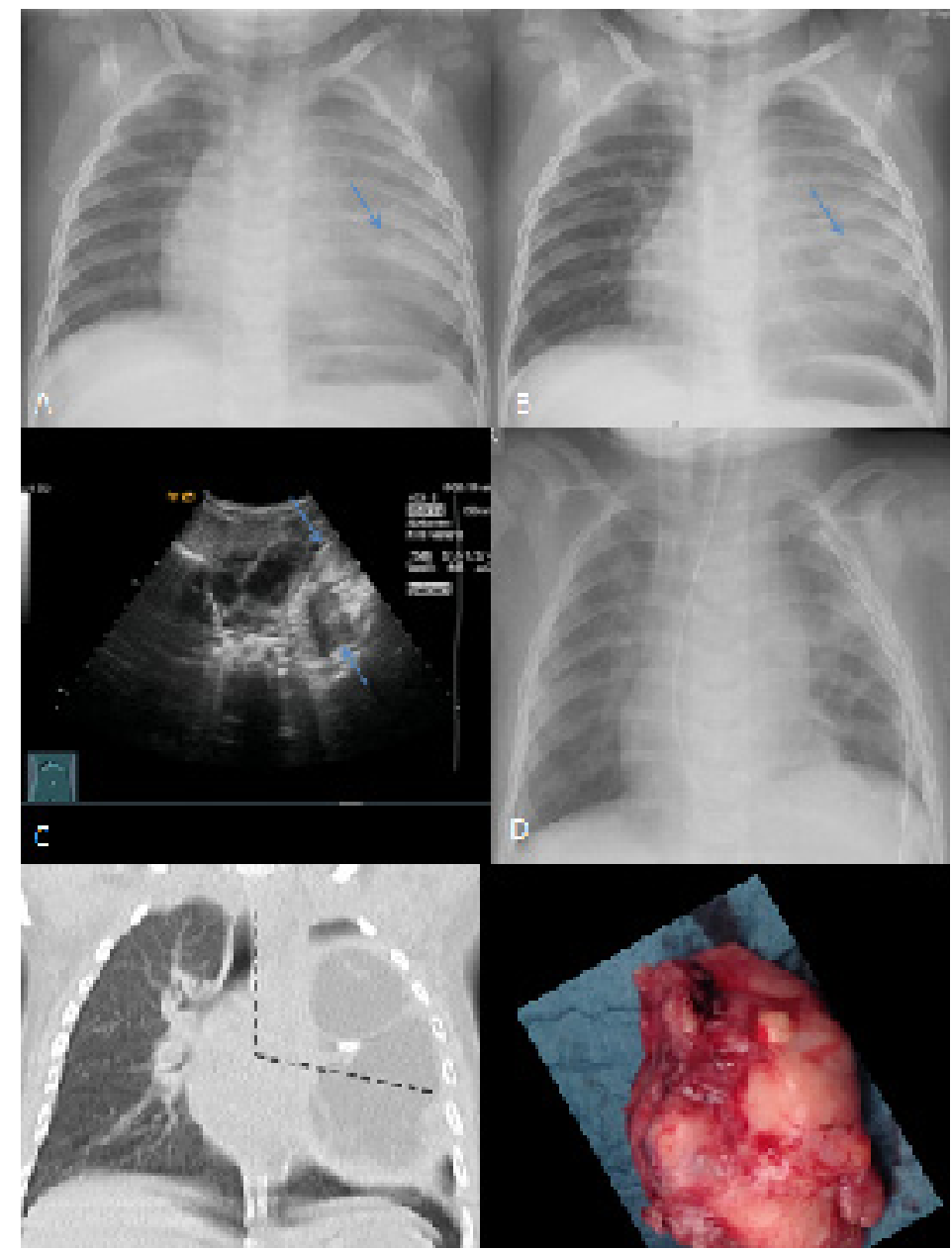

Figure 1: Depicts different radiologic and intraoperative images of the tumor

A) Depicts the chest X-ray on presentation with the radiological missed diagnosis of a bronchopneumonia. Note the fine calcifications in the illdefined mass (arrow)

B) Depicts the chest X-ray after 4 days of antibiotic treatment with an unchanged consolidation. Note the fine calcifications in the ill-defined mass (arrow)

C) Depicts ultrasound-scans preoperatively, which showed an unclear, paracardial consolidation with mixed hypo- and hyperechogenicity (arrows)

D) Depicts the chest X-ray immediately after thoracic surgery with a normalized aspect of the left lung. The chest drain in the left thoracic apparatus could be removed at the second postoperative day

E) Depicts a CT-scan preoperatively, which showed a large, well-defined heterogeneous mass (with areas of irregular nodularity, calcifications as well as fat attenuation along with soft tissue components) in the left hemithorax $(6.5 \times 4.5 \times 8 \mathrm{~cm})$, evolving in the anterior upper mediastinum and extending into the pleural cavity. The broken line marks the operative approach for tumor resection (hemiclamshell incision)

F) Depicts the completely resected teratoma 


\section{Discussion}

Teratomas account for up to $2 \%$ of childhood tumors and are seen in approximately 1 in 30,000 live births [4]. They originate from primitive cells and usually represent all of the three germ layers: endoderm, ectoderm and mesoderm. These tumors can be classified as mature (benign), intermediate and malignant. Most commonly, teratomas are found in sacrococcygeal, ovarial or testicular locations. The remaining locations (24\%) are in the mediastinum, the retroperitoneum and other midline structures [57]. Teratomas arising within the chest have been described to be located in the anterior or posterior mediastinum in the vicinity of the aorta, esophagus, pericardium, lung and diaphragm [5,7,8]. Other tumor entities such as manifestations of lymphomas, neuroblastomas, sarcomas or other kinds of small round blue cell tumors and metastases have to be excluded, as well as nonmalignant causes which mimicry a tumor as an infectious manifestation.

Mediastinal tumors and cysts often may cause no symptoms and are found on chest radiography, e.g. performed for symptoms of airway infections [9]. However, complications by obstruction, disruption or superinfections have been described [2,9,10]. Early diagnosis and resection therefore are important to avoid these complications. In our case the initial clinical presentation with persistent cough, signs of airway infection and the pathological lung auscultation together with the radiologic findings led first to the diagnosis of pneumonia, possibly due to Adenovirus, detected by PCR. However, the discrepancy between obvious clinical improvement, but unchanged pathology in the repeated chest X-rays does not fit with the working diagnosis of viral pneumonia. Therefore the diagnosis had to be reevaluated and differential diagnosis of pathologic auscultation and persistent cough had to be discussed. Therefore infectious causes as atypical pneumonia and pertussis had to be ruled out as well as a pleural effusion, congenital emphysema or malformation as well as a (benign or malign) tumor. The repeated X-ray led at last to the suspicion of an intrathoracic tumor, and additional confirmation by thoracic ultrasound and CT.

Complete surgical removal of the mediastinal teratoma is the curative treatment of choice. After complete resection of a mature teratoma, adjuvant therapy is not necessary and prognosis is usually excellent $[11,12]$. Even though recurrence or malignant transformations are unlikely in mature teratoma, follow-up examinations by radiography and tumor markers are recommended.

\section{Conclusion}

Our case highlights the need for careful evaluation by the treating physicians. If the chest X-ray provides a discrepancy to the clinical findings with persisting intrathoracic opacities, differential diagnosis should rule out intrathoracic or other malignant and non-malignant tumors with further diagnostics in order to prevent potential complications.

\section{Acknowledgement}

This publication was funded by the German Research Foundation (DFG) and the University of Wuerzburg in the funding programme Open Access Publishing.

\section{References}

1. Peterson CM, Buckley C, Holley S, Menias CO (2012) Teratomas: a multimodality review. Curr Probl Diagn Radiol 41: 210-9.

2. Reddy KRBK, Murthy GR, Sanjay KS (2013) Delayed diagnosis of infected posterior mediastinal teratoma. APSP J Case Rep 4: 23.

3. Comerci JT, Licciardi F, Bergh PA, Gregori C, Breen JL (1994) Mature cystic teratoma: a clinicopathologic evaluation of 517 cases and review of the literature. Obstet Gynecol 84: 22-8.

4. Senayli A, Deniz FE, Sezer T, Koseoglu RD, Senayli YA, et al. (2004) A case of bladder exstrophy and thoracal intramedullary teratoma. J Pediatr Surg 39: e9-11.

5. Lewis BD, Hurt RD, Payne WS, Farrow GM, Knapp RH, et al. (1983) Benign teratomas of the mediastinum. J Thorac Cardiovasc Surg 86: 727-31.

6. Dehner LP (1990) Germ cell tumors of the mediastinum. Semin Diagn Pathol 7: 266-84.

7. Gow KW, Koontz CS, Dickson P, Bannister L, Shehata B (2010) Retropleural teratoma presenting as an abdominal mass in a 9-month-old boy. J Pediatr Surg 45: e15-8.

8. Traubici J, Daneman A, Hayes-Jordan A, Fecteau A (2004) Primary germ cell tumor of the diaphragm. J Pediatr Surg 39: 1578-80.

9. Bhatt G, Nandan D, Sen A, Kanaujia P (2013) Fever of unknown origin: a case of post obstructive pneumonia complicating mature teratoma. Ann Med Health Sci Res 3: 461-3.

10. Meena GL, Jindal G, Rastogi R, Jindal K (2009) A 22-year-old woman with fever, shortness of breath and chest pain. Ann Thorac Med 4: 158-60.

11. Gatcombe HG, Assikis V, Kooby D, Johnstone PA (2004) Primary retroperitoneal teratomas: a review of the literature. J Surg Oncol 86: 107-13.

12. Göbel U, Calaminus G, Schneider DT, Koch S, Teske C, et al. (2006) The malignant potential of teratomas in infancy and childhood: the MAKEI experiences in non-testicular teratoma and implications for a new protocol. Klin Padiatr 218: 309-14. 


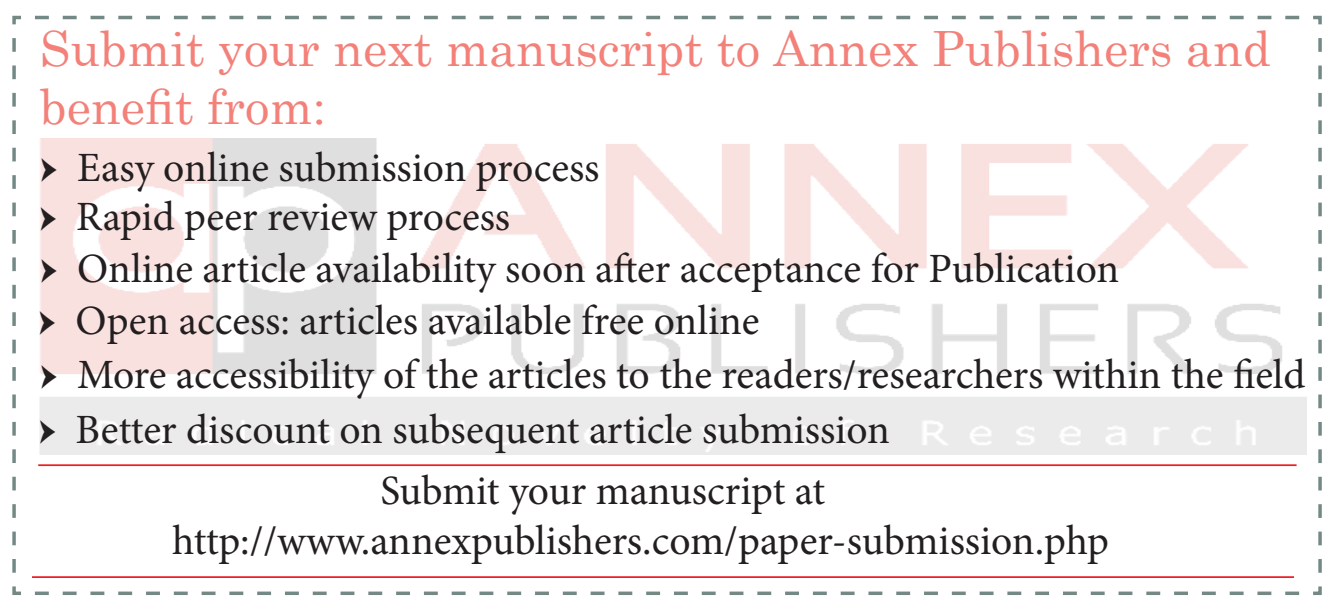

